\title{
Characterization of Deoxyribozymes that Synthesize Branched RNA
}

\author{
Yangming Wang and Scott K. Silverman*
}

Department of Chemistry, University of Illinois at Urbana-Champaign, 600 S. Mathews Ave., Urbana, Illinois 61801

Figures in this Supporting information are prefixed by the letter X (e.g., Figure X1) to distinguish them from those in the manuscript. All references cited by number are from the manuscript. See the manuscript's Experimental Section and refs. 6 and 9 for experimental details.

9F7 ligation assays with $\mathrm{Zn}^{2+}, \mathrm{Ni}^{2+}, \mathrm{Co}^{2+}$, and $\mathrm{Cd}^{2+}$

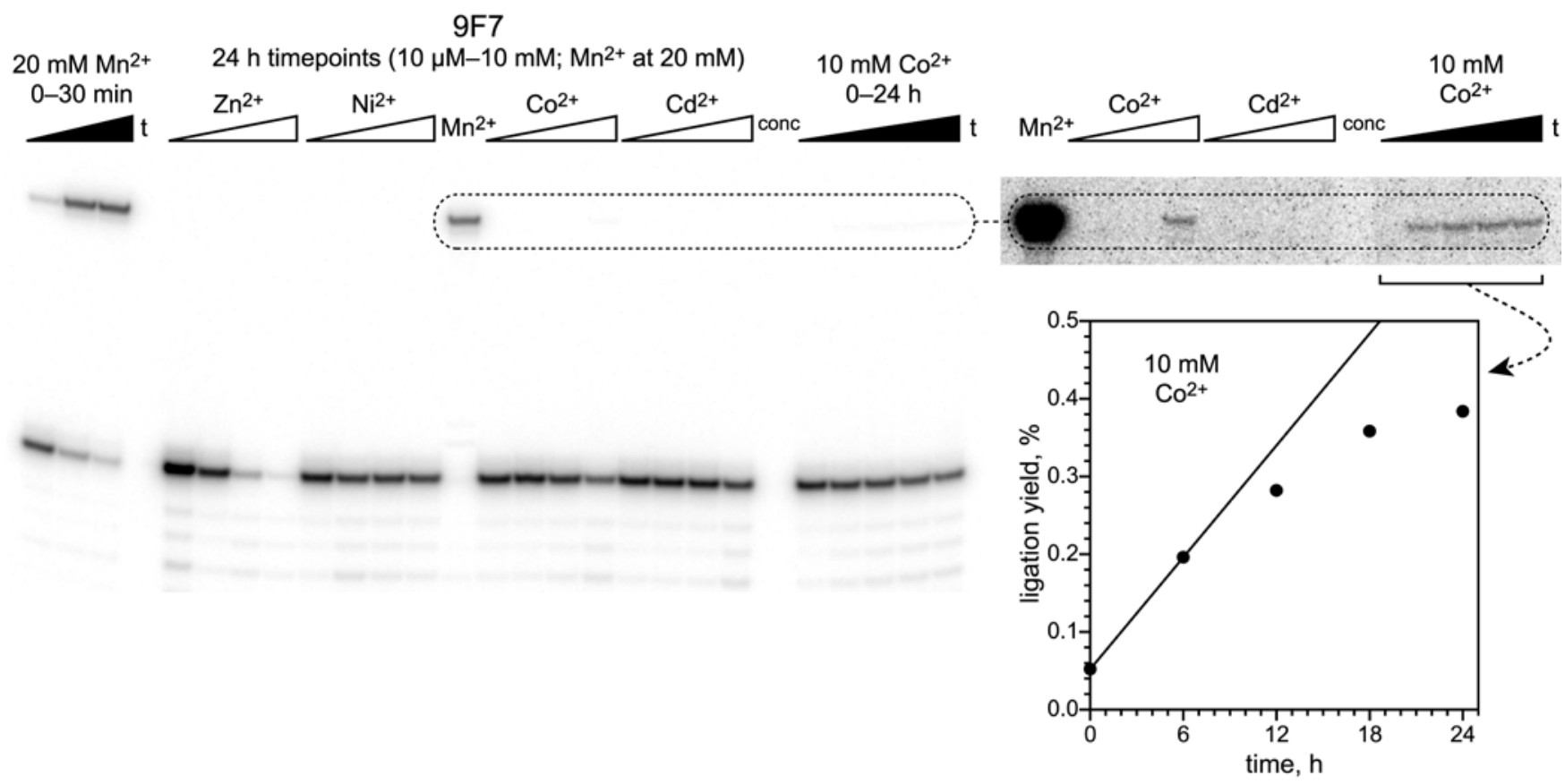

Figure X1. Assaying $\mathrm{Zn}^{2+}, \mathrm{Ni}^{2+}, \mathrm{Co}^{2+}$, and $\mathrm{Cd}^{2+}$ for the ability to support ligation activity by $9 \mathrm{~F} 7$. In the center portion of the gel image, the $\mathrm{Mn}^{2+}$ lane is a 24-h timepoint in $50 \mathrm{mM}$ HEPES, pH 7.5, $150 \mathrm{mM} \mathrm{NaCl}, 2 \mathrm{mM} \mathrm{KCl}$, and $20 \mathrm{mM} \mathrm{MnCl}_{2}$ at 37 ${ }^{\circ} \mathrm{C}$. For the other ions, 24-h timepoints are shown from similar reactions with $10 \mu \mathrm{M}, 100 \mu \mathrm{M}, 1 \mathrm{mM}$, or $10 \mathrm{mM}$ of the particular ion (chloride salt) instead of $\mathrm{Mn}^{2+}$. Degradation is evident with $10 \mathrm{mM} \mathrm{Zn}^{2+}$; there is significantly less degradation at shorter timepoints (e.g., $4 \mathrm{~h}$; data not shown). On the far left are timepoints $\left(0.5,5\right.$, and $30 \mathrm{~min}$ ) for $20 \mathrm{mM} \mathrm{Mn^{2+ }}$ as a control reaction. On the far right is an experiment with $0-24 \mathrm{~h}$ timepoints for $\mathrm{Co}^{2+}$ (the darker image is $200 \times$ relative to the lighter image). Some loss of signal at the longer timepoints is apparent, because the intensity of the unligated band at $24 \mathrm{~h}$ is only $50 \%$ of the same band's intensity at the zero timepoint. This is probably via hydrolysis of the 5 , $-{ }^{32} \mathrm{P}$-monophosphate, because no generic degradation "ladder" is visible at any of the timepoints. The initial slope of the plot of ligation yield versus time is $0.024 \%$ per hour $=4 \times 10^{-6} \mathrm{~min}^{-1}$, which is our best estimate of $k_{\mathrm{obs}}$ for $\mathrm{Co}^{2+}$; this is best described as an upper limit. With $k_{\text {obs }}$ for $\mathrm{Mn}^{2+}$ of $\sim 0.3 \mathrm{~min}^{-1}$, the relative rate for $\mathrm{Co}^{2+}$ is only about $10^{-5}$ that for $\mathrm{Mn}^{2+}$. The left-hand substrate was ...UA $\downarrow$ (uc) prepared by solid-phase synthesis. The purity of the $\mathrm{CoCl}_{2}$ was $99.998 \%$ (Alfa). See below for similar data for 9F21. 
$\underline{9 \mathrm{~F} 21 \text { ligation assays with } \mathrm{Zn}^{2+}, \mathrm{Ni}^{2+}, \mathrm{Co}^{2+} \text {, and } \mathrm{Cd}^{2+}}$

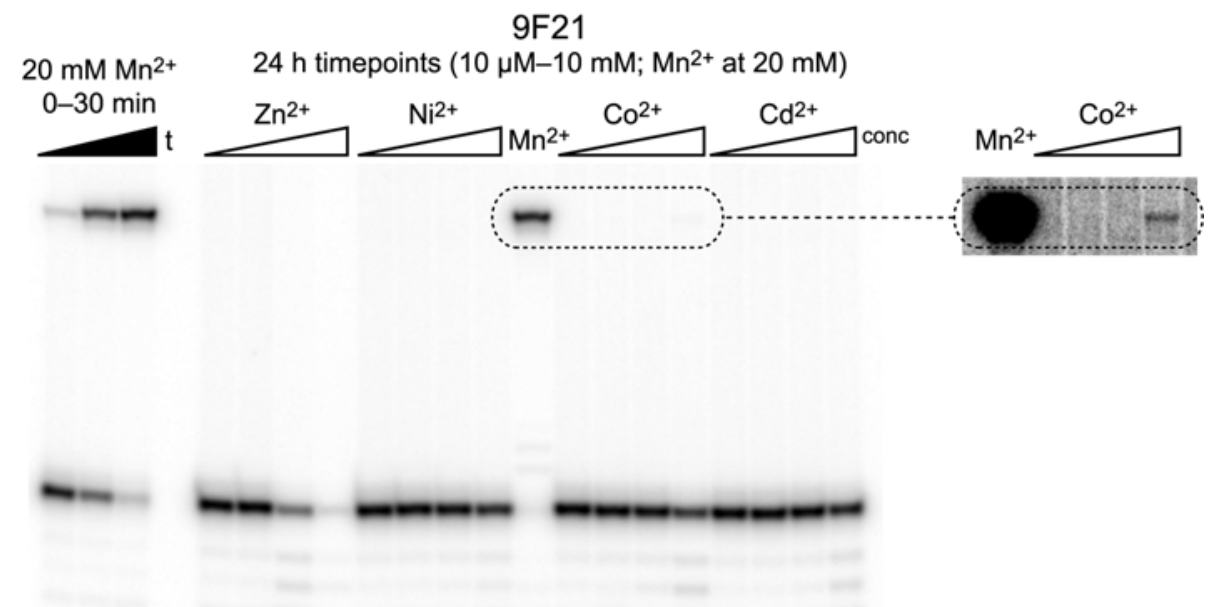

Figure X2. Assaying $\mathrm{Zn}^{2+}, \mathrm{Ni}^{2+}, \mathrm{Co}^{2+}$, and $\mathrm{Cd}^{2+}$ for the ability to support ligation activity by $9 \mathrm{~F} 21$. See Figure X1 for experimental details and similar results with 9F7.

\section{Longer timepoints for 9F7 and 9F21 ligation assays with varying L substrate sequences}

In Figure X3 are shown.longer timepoints for the experiments of Figure 4, in which the first three nucleotides of the left-hand substrate RNA were varied. These positions includes the key branch-site nucleotide ...CUA $\downarrow$ (ua). It is worthwhile to note that after $8 \mathrm{~h}$ incubation in $20 \mathrm{mM} \mathrm{Mn}^{2+}$, conditions under which RNA degradation is not necessarily problematic, 9F7 can create 2',5'-branched RNA where the branch site is any of $\mathrm{U}, \mathrm{C}, \mathrm{A}$, or $\mathrm{G}$ with potentially useful yields ( $\geq 20 \%$ in all cases).

\section{A $9 \mathrm{~F} 7$}

left-hand substrate (L): 5'-...CUA $\downarrow$ (ua)-3'
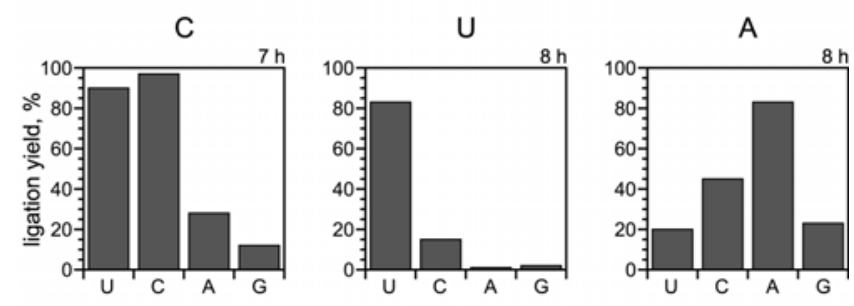

B

9F21

left-hand substrate (L): 5'-...CUA $\downarrow$ (ua)-3'
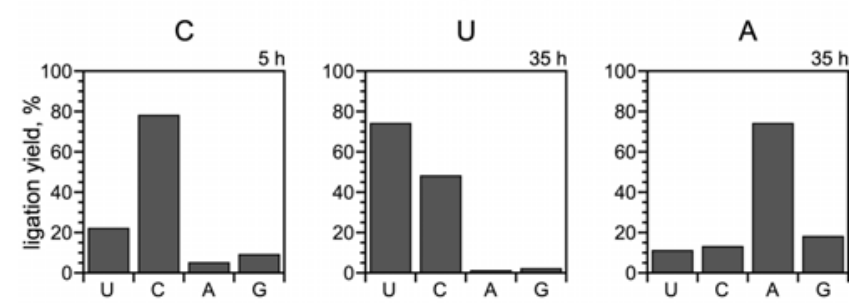

Figure X3. Longer timepoints for experiments of Figure 4 in which the CUA $\downarrow$ (ua) nucleotides are altered. These six panels may be compared directly with the analogous panels in Figure 4, for which data were taken after $0.5 \mathrm{~h}$ incubation. See the Figure 4 caption for details of the experiments. 
Testing NUA $\downarrow$ (ua) and $\downarrow$ GGAAN positions for Watson-Crick base pairing

Assays similar to those in Figure 4 were performed for RNA substrates of sequence NUA $\downarrow$ (ua) and (separately) $\downarrow$ GGAAN (Figure X4). These assays used 9F7 and 9F21 deoxyribozymes with all four possible DNA nucleotides (denoted Z) opposite the indicated RNA nucleotide (denoted X). Ligation yields are tabulated at short and long timepoints for each combination. The results show that WatsonCrick base pairs at these two positions support high ligation activity, with a pyrimidine (9F7) or C (9F21) required at the $X$ position in the left-hand substrate (Figure 4) and a purine favored at the $X$ position in the right-hand substrate (Figure 5). At the $\mathrm{X}-\mathrm{Z}$ positions, mismatches generally but not always have reduced activity compared to Watson-Crick matches.

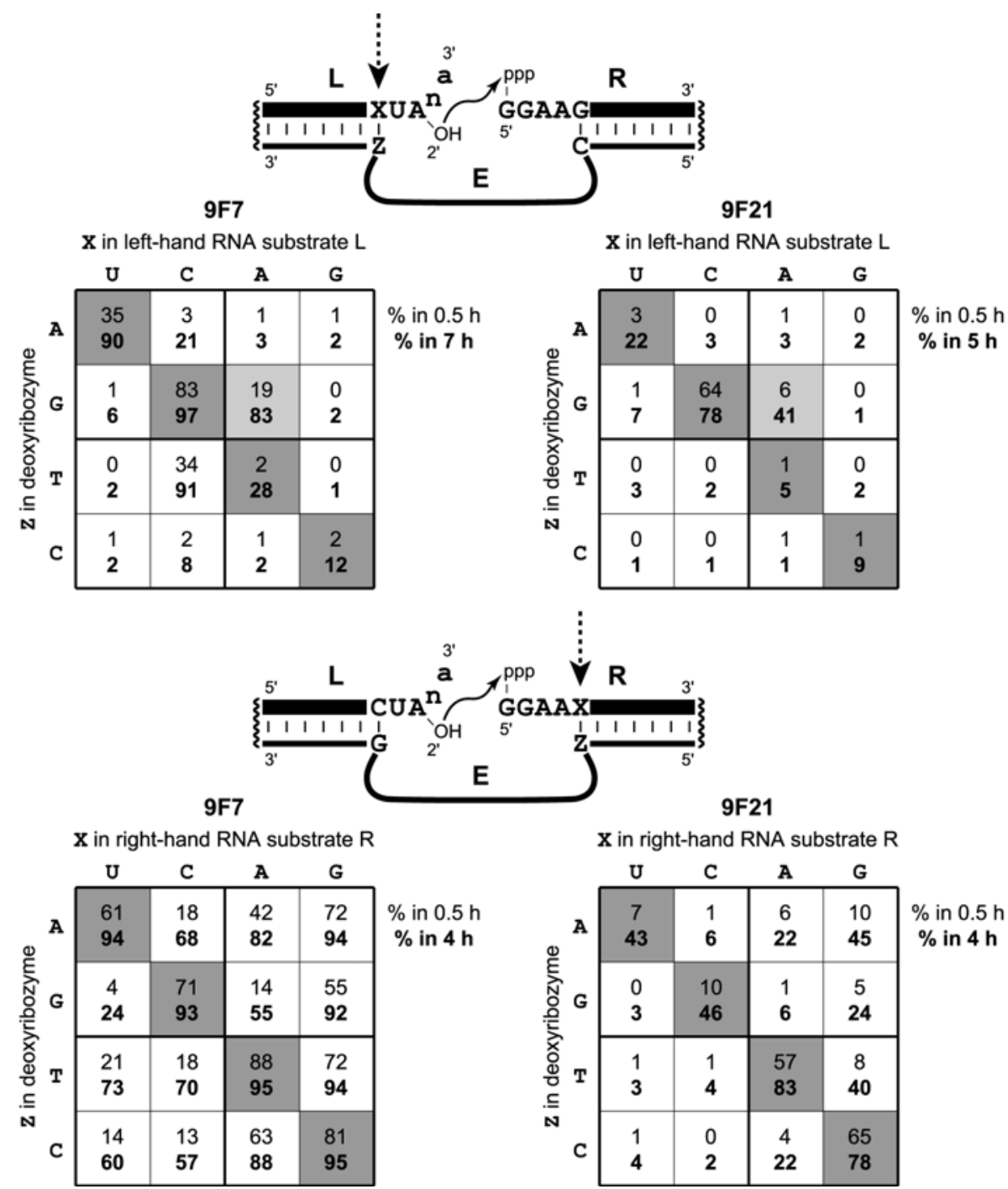

Figure X4. Testing for base pairing at two specific positions near the ligation site. For each of the 64 combinations of RNA substrates and deoxyribozyme (nucleotide $\mathrm{X}$ in RNA and nucleotide $\mathrm{Z}$ in DNA for the two positions in each of the two deoxyribozymes; $4 \times 4 \times 2 \times 2=64$ ), two timepoints were taken in addition to a 30 -second timepoint. The first additional timepoint was at $0.5 \mathrm{~h}$, and the second timepoint was at $4-7 \mathrm{~h}$ as indicated. The observed ligation yields at the two timepoints are shown in each box. Watson-Crick base pairs are shaded dark grey. The two A-G RNA-DNA combinations for the left-hand substrate for 9F7 and 9F21 are shaded light grey to highlight the anomaly that they provide higher ligation rate and yield than the Watson-Crick A-T combination. 
Testing a potential base-pairing interaction between the RNA and DNA in the left-hand binding arm
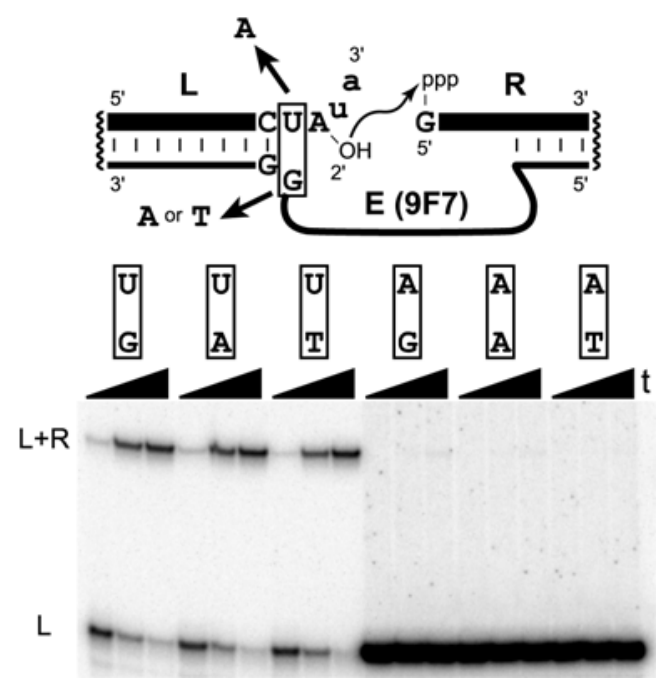

Figure X5. Testing for a Watson-Crick base pair between the left-hand RNA substrate nucleotide Y $\underline{\text { AA }} \downarrow$ and the final nucleotide of the 9F7 enzyme region. The boxed RNA nucleotide was either maintained as $U$ or changed to A, and the corresponding DNA nucleotide of 9F7 was changed from G to either A or T. The data indicate that a base pair is not formed between these two nucleotides. Assays were performed in $50 \mathrm{mM}$ HEPES, pH 7.5, $150 \mathrm{mM} \mathrm{NaCl}, 2 \mathrm{mM} \mathrm{KCl}$ and $20 \mathrm{mM}$ $\mathrm{MnCl}_{2}$ at $37^{\circ} \mathrm{C}$. Timepoints were at $0.5,5$, and $30 \mathrm{~min}$. The left-hand substrate was ...UA $\downarrow$ (ua) or ...AA $\downarrow$ (ua)prepared by transcription terminating with a 2',3'-diol. 


\section{Ligation by 9F7 to form linear 2'-5'-linked RNA using a short left-hand RNA substrate}

The data in Figure 7 demonstrate that 9F7 and 9F21 each create linear RNA from a "tail-less" lefthand substrate. For the 9F7 product, whether the new ligation junction is $3^{\prime}-5$ ' or 2' -5 ' was distinguished using the two complementary assays shown in Figure X6. These assays were described in detail in our previous manuscript (ref. 6). Briefly, an appropriate 8-17 deoxyribozyme selectively cleaves at the newly created $A \downarrow G$ junction if the linkage is $3^{\prime}-5^{\prime}$ (but not 2'-5'), whereas $100 \mathrm{mM}$ $\mathrm{Mg}^{2+}$ promotes selective cleavage of an RNA 2'-5' linkage (but not a 3'-5' linkage) at $\mathrm{pH} 9$ in the presence of the exactly complementary DNA. The results in Figure X6 for the 9F7 tail-less ligation product unambiguously indicate that this product is 2 '-5'-linked, consistent with our initial expectation. This product is formed by reaction of the same adenosine 2'-hydroxyl that reacts when the left-hand RNA substrate has a 3'-tail of any length greater than zero (i.e., when the adenosine 3'-hydroxyl is already participating in a conventional phosphodiester bond). Therefore, these data show that removing the 3'-tail does not change the site of reactivity, but this does lower the ligation rate considerably (compare Figures 9 and 10).

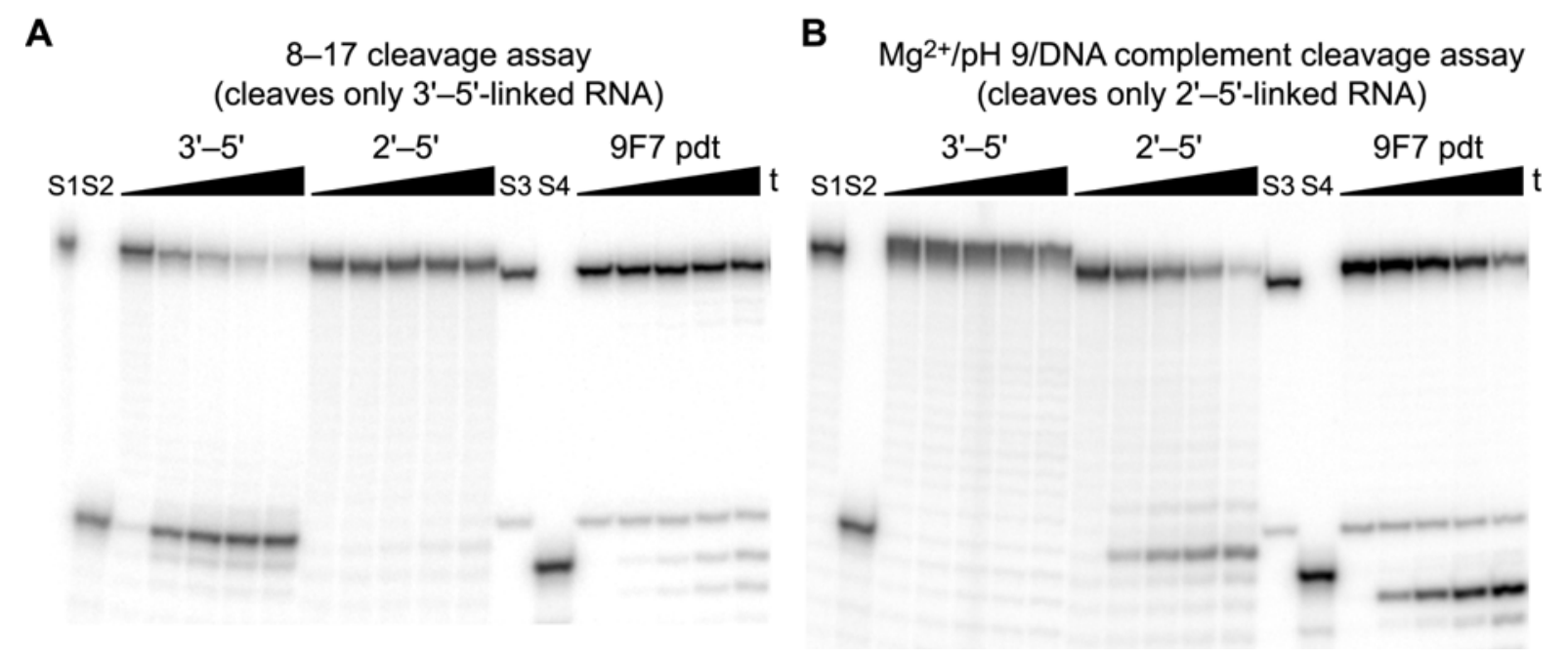

Figure X6. Assays to distinguish the 3'-5' versus 2'-5' nature of the linear 9F7 ligation product from the tail-less left-hand substrate. Both of these assays are described fully in ref. 6. (A) Using the 8-17 deoxyribozyme to reveal 3'-5' linkages. Timepoints at $0,20 \mathrm{~min}, 1,3$, and $6 \mathrm{~h}$. S1 is the 3'-5'-linked standard RNA from ref. 6 that is made from a left-hand subtrate $2 \mathrm{nt}$ longer than the tail-less substrate. S2 is the left-hand substrate standard ...UAUA terminating with a 2',3'cyclic phosphate (i.e., the expected product from $8-17$ cleavage of S1). S3 is the standard for the untreated $5{ }^{3}-{ }^{32} \mathrm{P}-$ radiolabeled 9F7 ligation product. S4 is the $5^{,}{ }_{-}^{32} \mathrm{P}$-radiolabeled tail-less left-hand RNA substrate standard. The timepoints marked 3' -5 ' show the expected cleavage of the 3' -5 '-linked standard RNA by $8-17$, whereas the timepoints marked 2' -5 ' show the expected lack of cleavage of the 2'-5'-linked standard RNA. The 9F7 ligation product shows essentially no cleavage by $8-17(<5 \%$; comparable to Figure $3 \mathrm{C}$ of ref. 6$)$. The trace impurity (lower band) in $\mathrm{S} 3$ and the $9 \mathrm{~F} 7 \mathrm{pdt}$ lanes is not identified. (B) Using $100 \mathrm{mM} \mathrm{Mg}^{2+}$ at $\mathrm{pH} 9$ in the presence of the exactly complementary DNA to reveal 2' -5 ' linkages. Timepoints at $0,2,6,12$, and $24 \mathrm{~h}$. Standards S1-S4 as in panel A. Note that S4 migrates slightly slower than the 9F7 pdt cleavage product as expected, because S4 has a 2',3'-diol terminus whereas the cleavage product has a $2^{\prime}, 3^{\prime}$-cyclic phosphate. The 9F7 ligation product shows the expected cleavage for a $2^{\prime}-5$ ' linkage with $\mathrm{t}_{1 / 2} \approx 11 \mathrm{~h}$ (ref. 6). 
9F21 is more sensitive than 9F7 to the left-hand substrate 3'-tail length

The data in Figure 8 demonstrate that 9F7 tolerates increasing the 3'-tail length with no reduction in yield and only a two-fold decrease in $k_{\mathrm{obs}}$. Here we show that 9F21 is more sensitive than 9F7 to the tail length. When the same set of left-hand substrates as in Figure 8 was tested with 9F21, the $k_{\text {obs }}$ was considerably lower for the longer substrates (Figure X7). While the $k_{\text {obs }}$ decreases by $\sim 40$-fold when the tail length is increased from 2 to 8 or $20 \mathrm{nt}$, the $k_{\text {obs }}$ is comparable $\left(\sim 0.003 \mathrm{~min}^{-1}\right)$ for both of the longer tail lengths. This suggests that 9F21 will accept even longer 3'-tails on left-hand RNA substrates.
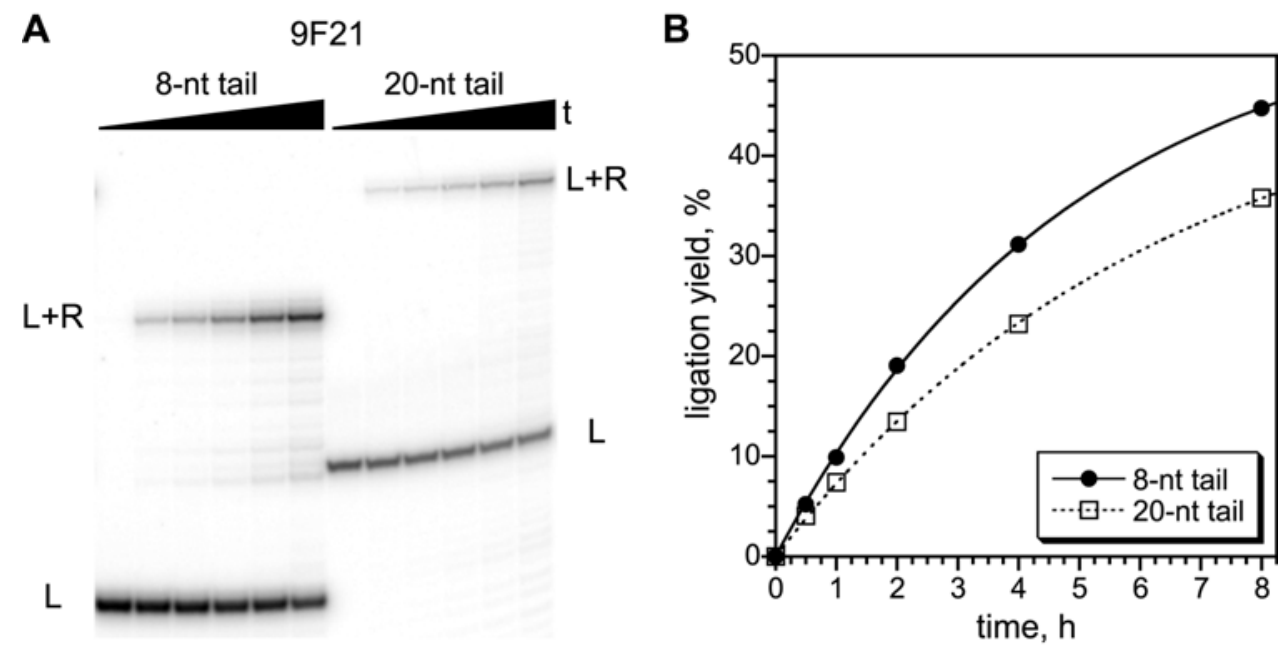

Figure X7. Testing 9F21 with longer left-hand RNA substrates. The experiment is essentially the same as that of Figure 8, with 9F21 instead of 9F7. (A) Gel image. (B) Kinetics plot. $k_{\text {obs }}$ values: 8-nt tail, $0.0034 \mathrm{~min}^{-1}$; 20 -nt tail, $0.0026 \mathrm{~min}^{-1}$. See Figure 1C for 9F21 with a 2-nt tail $\left(k_{\mathrm{obs}}=0.13 \mathrm{~min}^{-1}\right)$. 